\title{
Profiling of differentially expressed genes in adipose tissues of multiple symmetric lipomatosis
}

\author{
$\mathrm{KE} \mathrm{CHEN}^{1}$, LINGHAO WANG ${ }^{1}$, WENJUN YANG $^{1}, \mathrm{CHANGFA} \mathrm{WANG}^{2}, \mathrm{GUI}^{\mathrm{H}} \mathrm{HU}^{2}$ and $\mathrm{ZHAOHUI} \mathrm{MO}^{1}$ \\ Departments of ${ }^{1}$ Endocrinology and ${ }^{2}$ General Surgery, The Third Xiangya Hospital of Central South University, \\ Changsha, Hunan 410013, P.R. China
}

Received December 8, 2016; Accepted July 4, 2017

DOI: $10.3892 / \mathrm{mmr} .2017 .7437$

\begin{abstract}
Multiple symmetric lipomatosis (MSL) is a rare disorder characterized by aberrant multiple and symmetric subcutaneous adipose tissue accumulation in the face, neck, shoulders, back, chest and abdomen, severely affecting the quality of life of patients. At present, precise MSL etiology and pathogenesis remain to be elucidated. The present study first utilized a digital gene expression technique with a next-generation sequencing platform to profile differentially expressed genes in three cases of MSL vs. normal control tissue. cDNA libraries from these tissue specimens were constructed and DNA sequenced for identification of differentially expressed genes, which underwent bioinformatic analysis using the Gene Ontology (GO) enrichment, Kyoto Encyclopedia of Genes and Genomes (KEGG) and protein-protein interaction (PPI) network analyses. As a result, a total of 859 differentially expressed genes were identified, including 308 upregulated genes (C19orf80, Apelin, C21orf33, FAM166B and HSD11B2 were mostly upregulated 6.984-, 4.670-, 4.412-, 3.693- and 3.561-fold, respectively) and 551 downregulated genes [FosB proto-oncogene, AP-1 transcription factor subunit (FOSB), selectin (SEL) E, RAR related orphan receptor (ROR) B, salt inducible kinase (SIK)1 and epidermal grow th factor-like protein (EGFL)6 were mostly downregulated $-9.845,-8.243$, $-8.123,-7.702$ and -7.664 fold, respectively). The GO functional enrichment analysis demonstrated these differentially expressed genes were predominantly involved in biological processes and cellular components, while the KEGG pathway enrichment analysis demonstrated that ribosome, non-alcoholic fatty liver disease, human T-lymphotropic virus type 1 (HTLV-I) infection and Alzheimer's disease pathways were
\end{abstract}

Correspondence to: Professor Zhaohui Mo, Department of Endocrinology, The Third Xiangya Hospital of Central South University, 138 Tong Zi Po Road, Changsha, Hunan 410013, P.R. China

E-mail: easd04mzh@126.com

Key words: multiple symmetric lipomatosis, digital gene expression, differentially expressed genes, Gene Ontology, pathway, protein-protein interaction altered in MSL. The PPI network data demonstrated ubiquitin $\mathrm{C}$ (UBC), translocator protein (TSPO), Jun Proto-Oncogene, AP-1 Transcription Factor (JUN) and FOS were among these differentially expressed genes that participated in regulation of adipocyte differentiation, although no previous study has linked them to MSL. In conclusion, the present study profiled differentially expressed genes in MSL and identified gene pathways that may be associated with MSL development and progression.

\section{Introduction}

Multiple symmetric lipomatosis (MSL), first described in 1846 by Brodie (1), is a rare disorder characterized by aberrantly multiple, symmetric and substantial subcutaneous adipose tissue overgrowth in the face, neck, shoulders, back, chest and abdomen (2,3). MSL patients will present several clinical repercussions, including sleep apnea syndrome, swallowing and breathing difficulties, and polyneuropathies (4,5). MSL frequently occurs in men between 30 and 70 years of age with a high incidence rate of 1:25,000 in the Mediterranean region and a low rate in Asia. Alcoholism is a highly-associated risk factor in the development of MSL (6-8). MSL diagnosis is based on history, clinical appearance and X-ray computed tomography or magnetic resonance imaging. To date, the majority of studies of MSL have demonstrated that adipose tissue accumulation in MSL patients does not result in metabolic dysfunctions $(9,10)$, whereas other studies reveal an association of MSL with hyperlipidemia, diabetes mellitus, hypertension, or liver disease $(11,12)$. Clinically, there are no effective treatments for MSL, leading to a disappointing disease control, although other preventive measures or procedures, including alcohol withdrawal and weight reduction, may help in control of MSL fat mass, however do not reduce the progression of the disease (13). Currently, the primary treatment is surgical fat excision or liposuction and the latter is only effective on a smaller fat mass (14). The recurrence rate of surgical fat excision is $\sim 63 \%$, and the recurrence rate of liposuction is $95 \%$ (12).

At present, the precise MSL etiology and pathogenesis remain to be elucidated. Previous studies demonstrated an association of MSL with the point mutations (m.8344A $>\mathrm{G}$ or m.8363 G>A) in the mitochondrial DNA, genes of which are associated with the lipolytic pathway $(15,16)$. Further studies 
have demonstrated that MSL is associated with myoclonus epilepsy and ragged red fibers syndrome $(17,18)$. Furthermore, additional studies reported that pre-adipocytes express high levels of uncoupling protein 1 (UCP1) in MSL and the latter is a brown adipocyte marker for MSL adipocytes containing abundant mitochondria in the cytoplasm, suggesting overgrowth of MSL adipocytes from immature brown precursor cells $(19,20)$. In addition, defects in the adrenal $\beta 3$ adrenoceptor function or decrease in inducible nitric oxide synthase (iNOS) are also postulated in MSL since decrease in iNOS leads to reduction of nitric oxide and in turn, to enhancement of adipogenesis $(19,20)$. A recent study analyzed differential expression of p53 signaling pathway genes, nuclear receptors and co-regulators and mitochondrial genes using pathway cDNA arrays in adipose tissues derived stem cells in five sporadic MSL cases (21). Therefore, improved understanding of MSL pathogenesis and identification of gene alterations may aid the provision of novel strategies in MSL control, prevention and prognosis.

The present study employed a digital gene expression technique with a next-generation sequencing platform to first profile differentially expressed genes in MSL compared with normal control tissues. The technique overcomes the deficiency of cDNA microarray technique with improved accuracy, sensitivity, repeatability and detection range in analysis of gene transcripts. The present study aimed to provide novel information regarding the differentially expressed genes in MSL in order to identify novel strategies in prevention, treatment and pathogenesis of MSL in the future.

\section{Materials and methods}

Patients and samples collection. The present study recruited three male MSL patients (age range between 45 to 51 years old) who received medical treatment in The Department of Endocrinology and General Surgery, The Third Xiangya Hospital of Central South University (Changsha, China). These unrelated patients were clinically diagnosed with MSL and reported in the author's previous studies $(10,22)$. Clinically, these patients had fatty masses progressively appearing in the neck, upper arms, bilateral shoulders, upper thorax, back and abdomen with a history of heavy alcohol consumption (100-150, 100-150 and 150-200 $\mathrm{ml}$ of wine daily for 15,10 and 8 years, respectively). Furthermore, the present study obtained three age- and sex-matched control males from the outpatient clinic as control subjects. These control subjects had no history of diabetes, malignancies, acute infectious disease, or smoking history and were matched with MSL patients in terms of age, body mass index and where they lived. These samples were collected at The Third Xiangya Hospital, Central South University (Changsha, China) between July 2008 and September 2014. The present study was approved by the Human Ethical Review Committee of The Third Xiangya Hospital, Central South University and all studies were conducted according to the principles expressed in the Declaration of Helsinki. Subjects signed informed written consent prior to their inclusion in the present study. Tissue samples were obtained from the right upper abdomen subcutaneous lipomatous tissues of MSL patients and from the right upper abdomen subcutaneous adipose tissues of control subjects and then snap-frozen and stored at $-80^{\circ} \mathrm{C}$ until use.
RNA isolation, cDNA library construction and DNA sequencing. Tissue samples of each MSL and control subject were subjected to RNA isolation using TRIzol ${ }^{\circledR}$ (Invitrogen; Thermo Fisher Scientific, Inc., Waltham, MA, USA) according to the manufacturer's protocol. The concentration and purity of these RNA samples were then assessed using a Nano Photometer ${ }^{\circledR}$ spectrophotometer (Implen, Inc., Westlake Village, CA, USA) and a Qubit ${ }^{\circledR}$ RNA Assay kit in a Qubit ${ }^{\circledR}$ 2.0 Flurometer (Invitrogen; Thermo Fisher Scientific, Inc.), and RNA integrity was determined using the RNA Nano 6000 Assay kit (procedures were conducted according to the manufacturer's protocol) of the Bioanalyzer 2100 system (Agilent Technologies, Inc., Santa Clara, CA, USA).

A cDNA library was then constructed using Novogene Bioinformatics Technologies Co., Ltd. (Beijing, China) using a NEB Next ${ }^{\circledR}$ Ultra $^{\mathrm{TM}}$ RNA Library Prep kit for Illumina ${ }^{\circledR}$ (New England Biolabs, Inc., Ipswich, MA, USA) following the manufacturer's protocols. Briefly, mRNA was purified from the RNA samples using poly-T-oligo-attached magnetic beads and fragmented to $200 \mathrm{bp}$ using divalent cations under elevated temperature and then reversely transcribed into cDNA using random hexamer primers and M-MuLV Reverse Transcriptase. The second strand cDNA was synthesized using RNase H, dNTP and DNA polymerase I. Subsequently, polymerase chain reaction (PCR) was performed using Phusion High-Fidelity DNA polymerase, Universal PCR primers and Index (X) Primer for double-stranded cDNA amplification. The PCR products were then purified using the AMPure XP system and the library quality was assessed using the Agilent Bioanalyzer 2100 system and the cDNA library was sequenced using an Illumina Hiseq 2000/2500 platform and 100 bp/50 bp single-end reads were generated.

Data analysis. The raw data generated from Illumina Hiseq 2000 sequencing were processed through in-house Perl scripts. The processed data reads were filtered into clean reads by removing adaptor sequences, duplicated sequences, empty reads, or poly- $\mathrm{N}$ reads containing $>10 \%$ ' $\mathrm{N}$ ' and low-quality reads $(<\mathrm{Q} 20)$. The clean reads were then mapped to the reference sequences with using TopHat 2 available at www.ccb.jhu.edu/software/tophat.

Next, differentially expressed genes in MSL compared with controls were identified using the DESeq $\mathrm{R}$ package from www.bioinfo.au.tsinghua.edu.cn/software/degseq (23). DESeq provides statistical routines to determine differential gene expression with the digital gene expression data using a model based on the negative binomial distribution. The resulting P-values were then adjusted using the Benjamini and Hochberg's approach for controlling the false discovery rate (24). Genes with an adjusted $\mathrm{P}$-value $<0.05$ identified by DESeq were assigned as differentially expressed ones.

Bioinformatic analysis. These differentially expressed genes were grouped and analyzed using the Gene Ontology (GO) enrichment analysis with the GOseq $\mathrm{R}$ package (version 1.12.0), in which gene length bias was corrected. GO terms with corrected $\mathrm{P}<0.05$ were considered significantly enriched.

Subsequently, these differentially expressed genes were grouped into gene pathways using the pathway enrichment analysis with the Kyoto Encyclopedia of Genes and Genomes 
Table I. Primers used for reverse transcription-quantitative polymerase chain reaction.

\begin{tabular}{|c|c|c|}
\hline Gene name & Gene ID & Sequences of primers \\
\hline $\operatorname{PPAR} \gamma$ & NM_138711.3 & $\begin{array}{l}\text { 5'-ACCAAAGTGCAATCAAAGTGGA-3' } \\
\text { 5'-ATGAGGGAGTTGGAAGGCTCT-3' }\end{array}$ \\
\hline PDXK & NM_003681.4 & $\begin{array}{l}\text { 5'-GATTTGAGATTGACGCGGTGA-3' } \\
\text { 5'-CCCTCGTATAACCTGTGAGCAC-3' }\end{array}$ \\
\hline PGLS & NM_012088.2 & $\begin{array}{l}\text { 5'-CAGACTGCCGATCCCAGAAAG-3' } \\
\text { 5'-CCCTTGGAATGCCTGTCTCA-3' }\end{array}$ \\
\hline DGAT1 & NM_012079.5 & $\begin{array}{l}\text { 5'-TATTGCGGCCAATGTCTTTGC-3' } \\
\text { 5'-CACTGGAGTGATAGACTCAACCA-3' }\end{array}$ \\
\hline HOXC8 & NM_022658.3 & $\begin{array}{l}\text { 5'-ACCGGCCTATTACGACTGC-3' } \\
\text { 5'-TGCTGGTAGCCTGAGTTGGA-3' }\end{array}$ \\
\hline ATP5D & NM_001001975.1 & $\begin{array}{l}\text { 5'-TCCCACGCAGGTGTTCTTC-3' } \\
\text { 5'-GGAACCGCTGCTCACAAAGT-3' }\end{array}$ \\
\hline THY1 & NM_001311162.1 & $\begin{array}{l}\text { 5'-ATCGCTCTCCTGCTAACAGTC-3' } \\
\text { 5'-CTCGTACTGGATGGGTGAACT-3' }\end{array}$ \\
\hline SAR1A & NM_020150.4 & $\begin{array}{l}\text { 5'-ATAATGCAGGCAAAACCACTCT-3' } \\
\text { 5'-TGATGTCGGATGTAGTGTTGGAA-3' }\end{array}$ \\
\hline JUNB & NM_002229.2 & $\begin{array}{l}\text { 5'-ACGACTCATACACAGCTACGG-3' } \\
\text { 5'-GCTCGGTTTCAGGAGTTTGTAGT-3' }\end{array}$ \\
\hline RGS16 & NM_002928.3 & $\begin{array}{l}\text { 5'-ATCAGAGCTGGGCTGCGATA-3' } \\
\text { 5'-CAGGTCGAACGACTCTCTCC-3' }\end{array}$ \\
\hline HBEGF & NM_001945.2 & $\begin{array}{l}\text { 5'-ATCGTGGGGCTTCTCATGTTT-3' } \\
\text { 5'-TTAGTCATGCCCAACTTCACTTT-3' }\end{array}$ \\
\hline IGF1R & NM_001291858.1 & $\begin{array}{l}\text { 5'-TCGACATCCGCAACGACTATC-3' } \\
\text { 5'-CCAGGGCGTAGTTGTAGAAGAG-3' }\end{array}$ \\
\hline GAPDH & NM_001289746.1 & $\begin{array}{l}\text { 5'-GGCTGAGAACGGGAAGCTTGTCAT-3' } \\
\text { 5'-CAGCCTTCTCCATGGTGGTGAAGA-3' }\end{array}$ \\
\hline
\end{tabular}

PPAR- $\gamma$, peroxisome proliferator activated receptor; PDXK, pyridoxal pyridoxine, vitamin B6 kinase; PGLS, phosphogluconolactonase; DGAT1, diacylglycerol O-acyltransferase 1; HOXC8, homeobox C8; ATP5D, ATP synthase, $\mathrm{H}^{+}$transporting, mitochondrial F1 complex, $\Delta$ Subunit A; THY-1, Thy-1 cell surface antigen; SAR1A, secretion associated Ras Related GTPase 1A; IGF1R, insulin like growth factor 1 receptor, HBEGF, heparin binding EGF like growth factor; RGS16, regulator of G-protein signaling 16; JUNB, Jun Proto-Oncogene, AP-1 Transcription Factor B.

database (KEGG; www.genome.jp/kegg) and KOBAS version 2.0 software $(25,26)$. The statistical enriched differentially expressed genes in KEGG pathways were considered significant with corrected $\mathrm{P}<0.05$.

Protein-protein interaction (PPI) analysis was performed to predict PPIs of these differentially expressed genes (mips.helmholtz-muenchen.de/proj/ppi/). The protein network was then constructed using these differentially expressed gene IDs using the STRING database (http://string-db.org/) and Cytoscape version 3.5.1 software (http://www.cytoscape .org/download.html).

Reverse transcription-quantitative PCR (RT-qPCR). Total RNA was isolated from tissue samples using TRIzol reagent (Invitrogen; Thermo Fisher Scientific, Inc.) and $1 \mu \mathrm{g}$ of each RNA sample was reversely transcribed into cDNA using a reverse transcription kit (Fermentas; Thermo Fisher Scientific, Inc., Pittsburgh, PA, USA). PCR amplification was performed for 12 genes that were randomly selected for validation of gene profiling data on a Mastercycler ${ }^{\circledR} \mathrm{ep}$ realplex real-time PCR (Eppendorf, Hamburg, Germany). The primer pairs are listed in Table I and were synthesized by Shanghai Shenggong Biology Engineering Technology Service, Ltd. (Shanghai, China). GAPDH was used as an endogenous control. The SYBR-Green PCR assay contained $10 \mu \mathrm{l}$ of QuantiTect SYBR-Green PCR Master mix (ToYoBo, Osaka, Japan), $2 \mu \mathrm{l}$ of cDNA, $1.2 \mu \mathrm{l}$ of each primer $(10 \mu \mathrm{M})$ and $6.8 \mu \mathrm{l}$ of RNase-free water, The cycling conditions were as follows: An initial denaturation at $95^{\circ} \mathrm{C}$ for $1 \mathrm{~min}$, followed by 40 cycles of denaturation for $15 \mathrm{sec}$ at $95^{\circ} \mathrm{C}$, annealing for $30 \mathrm{sec}$ at $60^{\circ} \mathrm{C}$ and extension for $30 \mathrm{sec}$ at $72^{\circ} \mathrm{C}$. Levels of the relative gene expression were computed using the $2^{-\Delta \Delta \mathrm{CT}}$ method (27), and each experiment contained three replicates.

Statistical analyses. All statistical analyses were performed by using SPSS software version 13.0 (SPSS, Inc., Chicago, IL, USA) and an unpaired Student's t-test was used to analyze data between MSL and control. $\mathrm{P}<0.05$ was considered to indicate a statistically significant difference. 
Table II. Sequence statistics of MSL and control.

\begin{tabular}{lccccccc}
\hline Sample ID & Raw reads & Clean reads & Clean bases & Error rate $(\%)$ & Q20 (\%) & Q30 (\%) & GC content (\%) \\
\hline A1 & $32,904,914$ & $31,084,596$ & $3.89 \mathrm{G}$ & 0.01 & 96.71 & 92.87 & 51.46 \\
A2 & $28,879,428$ & $27,757,944$ & $3.47 \mathrm{G}$ & 0.01 & 96.82 & 92.95 & 54.0 \\
A3 & $34,129,132$ & $32,691,812$ & $4.09 \mathrm{G}$ & 0.01 & 96.88 & 93.11 & 52.37 \\
B1 & $27,662,982$ & $26,610,062$ & $3.33 \mathrm{G}$ & 0.01 & 96.72 & 92.76 & 52.68 \\
B2 & $33,834,004$ & $32,320,326$ & $4.04 \mathrm{G}$ & 0.01 & 96.69 & 92.78 & 53.7 \\
B3 & $42,327,474$ & $38,225,640$ & $4.78 \mathrm{G}$ & 0.01 & 97.72 & 94.34 & 51.92 \\
\hline
\end{tabular}

Q20 and Q30, Phred scores representing the sequencing error rates of 0.01 and 0.001, respectively; Qphred=-10log $\log _{10}(\mathrm{e})$. MSL, multiple symmetric lipomatosis.

Table III. Expression of the top twenty upregulated genes in MSL compared with control, isolated from abdomen adipose tissue.

\begin{tabular}{|c|c|c|c|}
\hline Gene_ID & Gene name & $\log _{2}$ (Fold change) & P-value \\
\hline ENSG00000130173 & C19orf80 & 6.984 & $7.07 \times 10^{-09}$ \\
\hline ENSG00000171388 & APLN & 4.670 & $7.06 \times 10^{-07}$ \\
\hline ENSG00000160221 & $\mathrm{C} 21$ orf 33 & 4.412 & $9.60 \times 10^{-09}$ \\
\hline ENSG00000215187 & FAM166B & 3.693 & $4.14 \times 10^{-08}$ \\
\hline ENSG00000176387 & HSD11B2 & 3.561 & $2.51 \times 10^{-240}$ \\
\hline ENSG00000170522 & ELOVL6 & 3.289 & $8.86 \times 10^{-06}$ \\
\hline ENSG00000142583 & SLC2A5 & 3.286 & $1.53 \times 10^{-08}$ \\
\hline ENSG00000130208 & APOC1 & 3.272 & $1.86 \times 10^{-05}$ \\
\hline ENSG00000132170 & $\operatorname{PPAR} \gamma$ & 3.180 & $1.22 \times 10^{-4}$ \\
\hline ENSG00000186188 & FFAR4 & 3.040 & $1.913 \times 10^{-4}$ \\
\hline ENSG00000100344 & PNPLA3 & 2.860 & $4.05 \times 10^{-14}$ \\
\hline ENSG00000072310 & SREBF1 & 2.859 & $9.14 \times 10^{-42}$ \\
\hline ENSG00000164308 & ERAP2 & 2.809 & $2.08 \times 10^{-4}$ \\
\hline ENSG00000123080 & CDKN2C & 2.734 & $1.58 \times 10^{-06}$ \\
\hline ENSG00000236824 & BCYRN1 & 2.632 & $1.70 \times 10^{-11}$ \\
\hline ENSG00000224940 & PRRT4 & 2.617 & $2.57 \times 10^{-19}$ \\
\hline ENSG00000087237 & CETP & 2.567 & $5.41 \times 10^{-06}$ \\
\hline ENSG00000151365 & THRSP & 2.519 & $1.84 \times 10^{-56}$ \\
\hline ENSG00000062282 & DGAT2 & 2.494 & $9.13 \times 10^{-06}$ \\
\hline ENSG00000230630 & DNM3OS & 2.426 & $4.93 \times 10^{-70}$ \\
\hline
\end{tabular}

MSL, multiple symmetric lipomatosis.

\section{Results}

DNA sequencing of cDNA libraries from MSL and control. To profile differentially expressed genes in MSL and explore genes that may associate with MSL pathogenesis, 6 cDNA libraries ( 3 of MSL A1, A2 and A3 vs. 3 of control B1, B2 and B3) were constructed. Following the removal of adaptor sequences, duplicated sequences, poly- $\mathrm{N}$ reads containing $>10 \%$ ' $\mathrm{N}$ ' and low-quality reads $(<\mathrm{Q} 20)$, a total number of clean reads per library ranged between 26.6 and 38.2 million were obtained. The Q20 values were $>96 \%$ and GC content $\sim 53 \%$ (Table II).

Identification of differentially expressed genes in MSL. The present study obtained a total of 859 genes that were differentially expressed in MSL, of which 308 genes were upregulated and 551 were downregulated (the top 20 up- and downregulated genes are presented in Tables III and IV). Specifically, expression levels of C19orf80, apelin (APLN), C21orf33, FAM166B and HSD11B2 were the most upregulated in MSL, whereas expression levels of FosB proto-oncogene, AP-1 transcription factor subunit (FOSB), selectin (SEL) E, RAR related orphan receptor (ROR) $B$, salt inducible kinase (SIK)1 and epidermal growth factor-like protein (EGFL) were the most downregulated. These differentially expressed genes were then clustered together in Fig. 1, where the red colored genes are upregulated and the blue colored genes downregulated. The results verified that MSL vs. the normal control had differentially expressed genes. 
Table IV. Expression of the top twenty downregulated genes in MSL compared with control, isolated from abdomen adipose tissues.

\begin{tabular}{llcl}
\hline Gene_ID & Gene Name & Log $_{2}($ Fold change $)$ & P-value \\
\hline ENSG00000125740 & FOSB & -9.845 & $2.67 \times 10^{-36}$ \\
ENSG00000007908 & SELE & -8.243 & $1.00 \times 10^{-09}$ \\
ENSG00000198963 & RORB & -8.123 & $1.365 \times 10^{-4}$ \\
ENSG00000142178 & SIK1 & -7.702 & $3.94 \times 10^{-07}$ \\
ENSG00000198759 & EGFL6 & -7.664 & $2.06 \times 10^{-08}$ \\
ENSG00000120738 & EGR1 & -7.466 & $1.90 \times 10^{-06}$ \\
ENSG00000122877 & EGR2 & -7.397 & $1.65 \times 10^{-27}$ \\
ENSG00000184557 & SOCS3 & -6.572 & $3.17 \times 10^{-30}$ \\
ENSG00000073756 & PTGS2 & -6.192 & $4.95 \times 10^{-12}$ \\
ENSG00000162772 & ATF3 & -6.104 & $7.19 \times 10^{-05}$ \\
ENSG00000136244 & IL6 & -6.085 & $2.64 \times 10^{-42}$ \\
ENSG00000123358 & NR4A1 & -5.894 & $5.48 \times 10^{-59}$ \\
ENSG00000170345 & FOS & -5.578 & $1.88 \times 10^{-10}$ \\
ENSG00000183186 & C2CD4C & -5.572 & $4.32 \times 10^{-15}$ \\
ENSG00000172602 & RND1 & -5.462 & $3.90 \times 10^{-07}$ \\
ENSG00000128016 & ZFP36 & -5.381 & $1.23 \times 10^{-4}$ \\
ENSG00000108342 & CSF3 & -5.324 & $1.65 \times 10^{-05}$ \\
ENSG00000144802 & NFKBIZ & -5.060 & $3.47 \times 10^{-07}$ \\
ENSG00000179388 & EGR3 & -5.047 & $3.50 \times 10^{-22}$ \\
ENSG00000171223 & JUNB & -4.924 & $2.28 \times 10^{-08}$ \\
\hline
\end{tabular}

MSL, multiple symmetric lipomatosis.

A volcano map (Fig. 2) with $\log _{2}$ (fold change) as the abscissa and $-\log _{10}$ (q-value) as the ordinate was used to demonstrate the overall distribution of differentially expressed genes between MSL and controls. In this volcano map, upregulated genes are presented as red dots and downregulated genes as green dots.

Functional annotation and pathway assignment. GO term analysis was then performed to group genes with similar functions and associations, and KEGG was used to group these differentially expressed genes into gene pathways. These differentially expressed genes could be enriched into 8,162 GO categories for different biological processes, cellular components and molecular functions. As presented in Fig. 3, the top $30 \mathrm{GO}$ categories for 26 different biological processes and 4 cellular components were identified. Of these $30 \mathrm{GO}$ categories, cellular component 'cytoplasm' was the most enriched, involving 584 differentially expressed genes. Of the 26 biological processes, the GO categories 'positive regulation of biological process', 'positive regulation of cellular process' and 'developmental process' were most enriched.

The KEGG pathway database analysis of these differentially expressed genes enriched 245 gene pathways and Fig. 4 demonstrates the top 20 pathways, in which 27 differentially expressed genes were enriched 'ribosome', 26 differentially expressed genes in 'non-alcoholic fatty liver disease (NAFLD)', 33 differentially expressed genes in 'HTLV-I infection' and 26 differentially expressed genes in Alzheimer's disease (Fig. 4).
Protein-protein interaction analysis. Subsequently, protein-protein interaction analysis of these differentially expressed genes was performed. In Fig. 5, 35 genes were demonstrated to be linked by more than 40 directed edges, of which ubiquitin C (UBC), translocator protein (TSPO), Jun Proto-Oncogene, AP-1 Transcription Factor (JUN) FOS and EGFR were most enriched with neighbor proteins.

RT-qPCR confirmation of differentially expressed genes in MSL tissues. The expression of 12 randomly selected differentially expressed genes in MSL was next verified using RT-qPCR. The data confirmed that peroxisome proliferator activated receptor (PPAR) $-\gamma$, pyridoxal pyridoxine, vitamin B6 kinase, phosphogluconolactonase, diacylglycerol O-acyltransferase 1 , homeobox $\mathrm{C} 8$, ATP synthase, $\mathrm{H}^{+}$transporting, mitochondrial F1 complex, D Subunit A, Thy-1 cell surface antigen and secretion associated Ras Related GTPase 1A were indeed significantly upregulated in MSL compared with control. Conversely, insulin like growth factor 1 receptor, heparin binding EGF like growth factor, regulator of G-protein signaling 16 and JUNB were significantly downregulated in MSL (Fig. 6), indicating that these data are highly consistent with the gene profiling data.

\section{Discussion}

The current study first profiled differentially expressed genes in MSL compared with normal controls and identified a total of 859 differentially expressed genes in MSL, of which 308 genes 


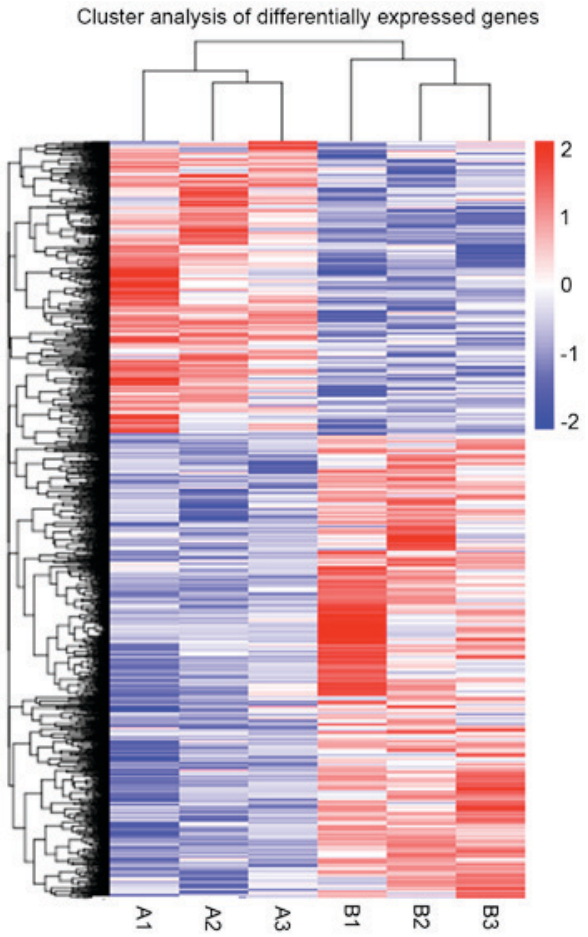

Figure 1. Hierarchical cluster of differentially expressed genes. Hierarchical cluster analysis was conducted for differential expression of genes using a fold change $<0.5$ or $>2$ and $\mathrm{P}<0.05$. The relative levels of differentially expressed genes are depicted to the color scale. Blue represents low level, white for medium level and red for high level. Each row indicates a gene and each column represents a tissue sample. A1, A2 and A3 represent MSL samples, and B1, B2 and B3 are normal samples. MSL, multiple symmetric lipomatosis.

were upregulated and 551 downregulated. The most upregulated genes in MSL were C19orf80, APLN, C21orf33, FAM166B and HSD11B2, and the most downregulated genes were FOSB, SELE, RORB, SIK1 and EGFL6. The current study profiled differentially expressed genes in MSL and identified gene pathways that may be associated with MSL development and progression. The data from the current study were partly consistent with those of a previous study reported by Harsch et al (28) using ELISA to detect decreased interleukin (IL)6 and increased leptin (LEP) in plasma, however are quite different from those of Prantl et al (21) which demonstrated that levels of IL6 and tumor necrosis factor (TNF) were upregulated, as the data from the current study demonstrated downregulated IL6 and TNF levels. The data from the current study also failed to demonstrate upregulated levels of UCP1, 2, 3 and 4. Future studies will further investigate the role of these differentially expressed genes and gene pathways in MSL development and progression.

The current study grouped and enriched these differentially expressed genes into functional groups of genes using GO categories and KEGG pathway analyses. The data demonstrated that the 'cytoplasm' was the most enriched category in GO term analysis. The cell cytoplasm is a component where the majority of gene products are localized, suggesting these genes may take part in MSL progression in the cytoplasm. Other GO terms were the 'positive regulation of biological process', 'positive regulation of cellular process' and 'developmental process'. The development of the cardiovascular symptom is highly associated with fat accumulation $(29,30)$,

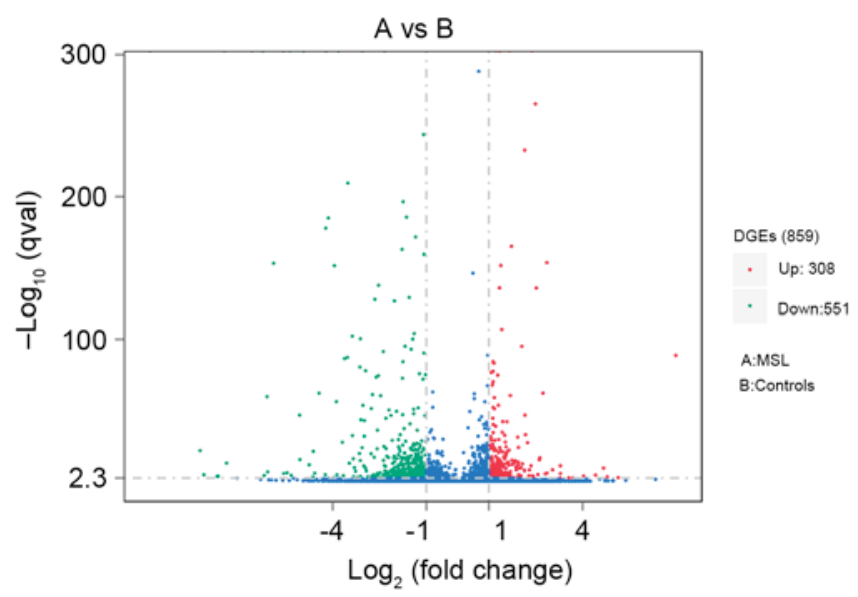

Figure 2. Volcano plot analysis. The detected genes are presented in the volcano plot using $\log _{2}$ (fold change) as $\mathrm{x}$-axis and $-\log _{10}$ (P-value) as $\mathrm{y}$-axis. The upregulated genes in MSL compared with controls, are presented in red, whereas the downregulated genes are in green. Genes without significant diversity between MSL and control are presented in blue. DGE, digital gene expression; MSL, multiple symmetric lipomatosis.

suggesting that these differentially expressed genes may regulate cellular process, particularly adipocyte development. In pathway analysis, the 'ribosome' was most enriched, followed by 'NAFLD', 'HTLV-I infection' and 'Alzheimer's disease'. The ribosome, the site of protein translation, is formed by ribosome proteins and RNA, and localized in the cytoplasm. The present study identified 27 differentially expressed genes that are ribosome proteins, although there is no study, to the best of the authors' knowledge, that reports the correlation of MSL with the ribosome protein, suggesting that these 27 ribosome proteins may regulate the translation of adipocytokines. There is also no direct association between NAFLD and MSL in the literature, however NAFLD pathogenesis is involved in the adipocytokine signaling to promote adipogenesis, suggesting that NAFLD and MSL may utilize similar gene pathways. Protein-protein interaction analysis identified that UBC was in the hub of the network and formed a sub-network by directly connecting to 447 other proteins (Fig. 5), indicating that UBC serves a role in MSL development and progression. However, Prantl et al (21) did not provide data on UBC, TSPO, JUN, FOS and EGFR, whereas the present study did not find alterations in the p53 signaling pathway, nuclear receptors and co-regulators or mitochondrial genes.

UBC protein is a 76-amino acid polypeptide expressed in all eukaryotes; it is a polyubiquitin precursor that functions to maintain normal cellular ubiquitin levels under stress conditions (31). Ubiquitin may ubiquitinate and modify cellular proteins to alter protein levels, functions, localization or fate $(32,33)$. Polyubiquitin-C serves a crucial role in regulation of protein turnover, particularly under stress conditions $(31,32)$. Furthermore, the ubiquitin-proteasome system degrades short-lived regulatory proteins and long-lived structural proteins (33). Once poly-ubiquitinated, proteins are undergo degradation through the $26 \mathrm{~S}$ proteasomes. A previous study demonstrated that accumulation of poly-ubiquitinated proteins induced by all $3 \omega-3$ polyunsaturated fatty acids in adipocytes promotes the degradation of fatty acid synthase and blocks adipogenesis (34). The current study identified that 


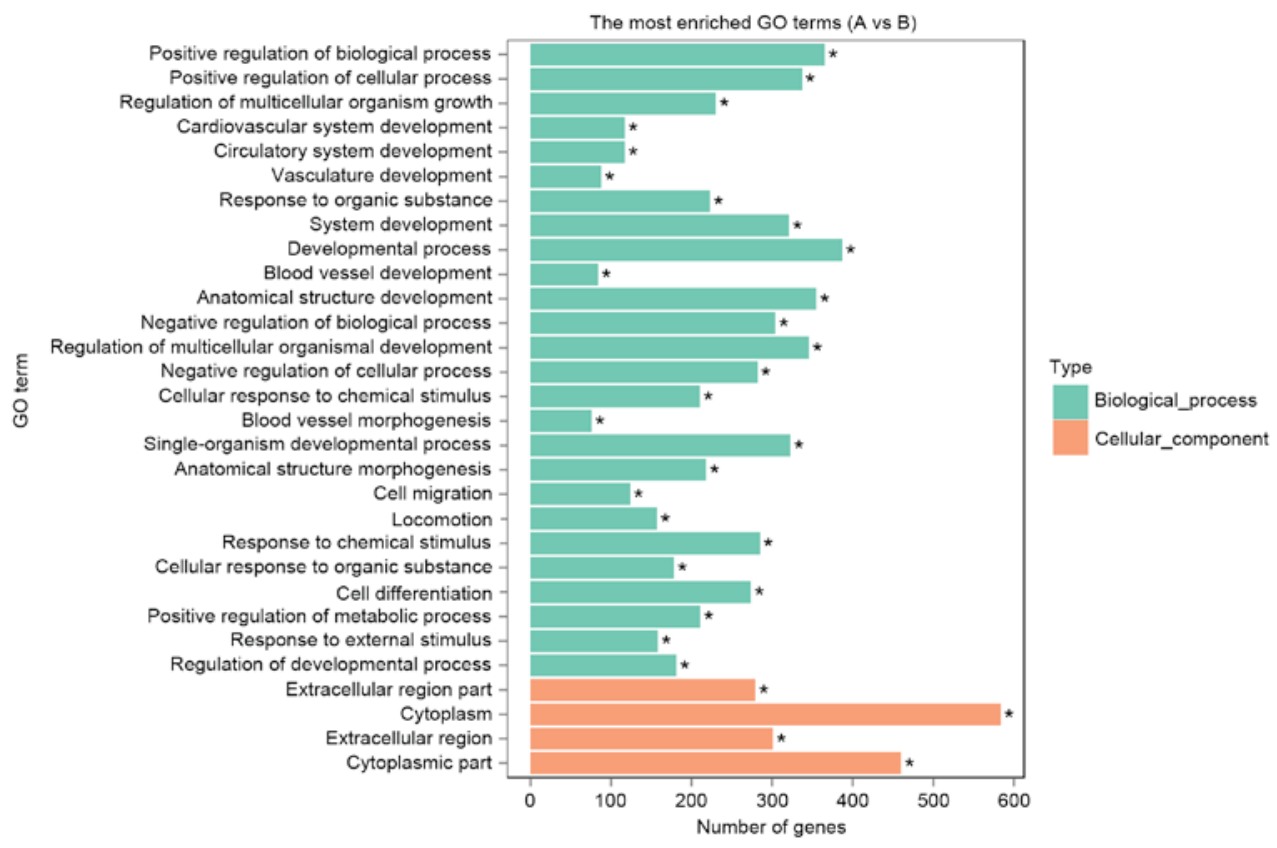

Figure 3. GO terms of the top 30 enriched genes. The GO enrichment analysis grouped these differentially expressed genes into functional groups. The green column represents biological processes and the orange column represents cellular components. GO, Gene Ontology.

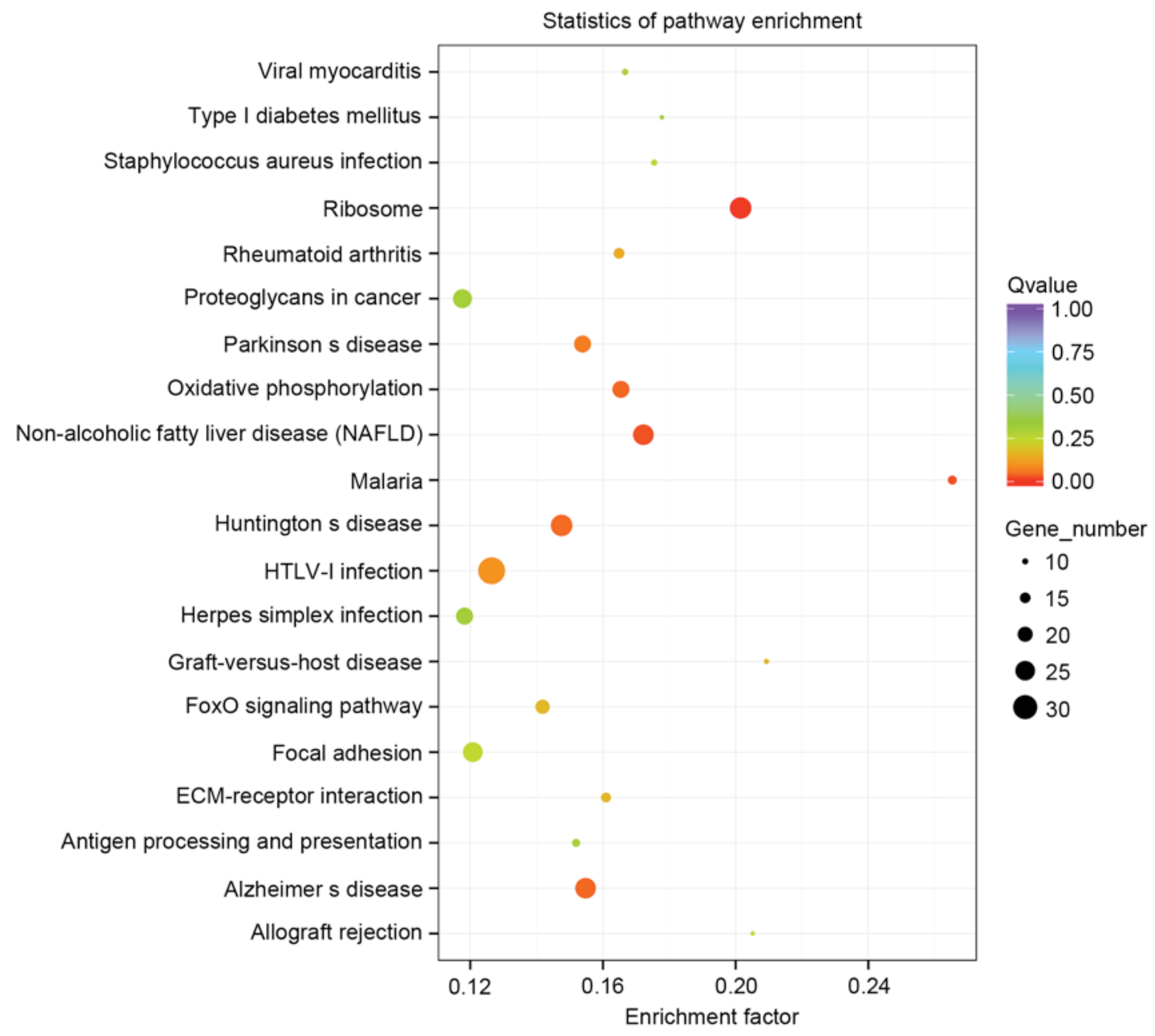

Figure 4. KEGG pathway analysis of the top $20 \mathrm{KEGG}$ enriched gene pathway-related diseases. These differentially expressed genes were grouped into gene pathways using the pathway enrichment analysis with the KEGG database and KOBAS software. Low q-values are in red and high q-values are in blue, the size of the circle is proportional to the number of enriched genes. KEGG, Kyoto Encyclopedia of Genes and Genomes. 


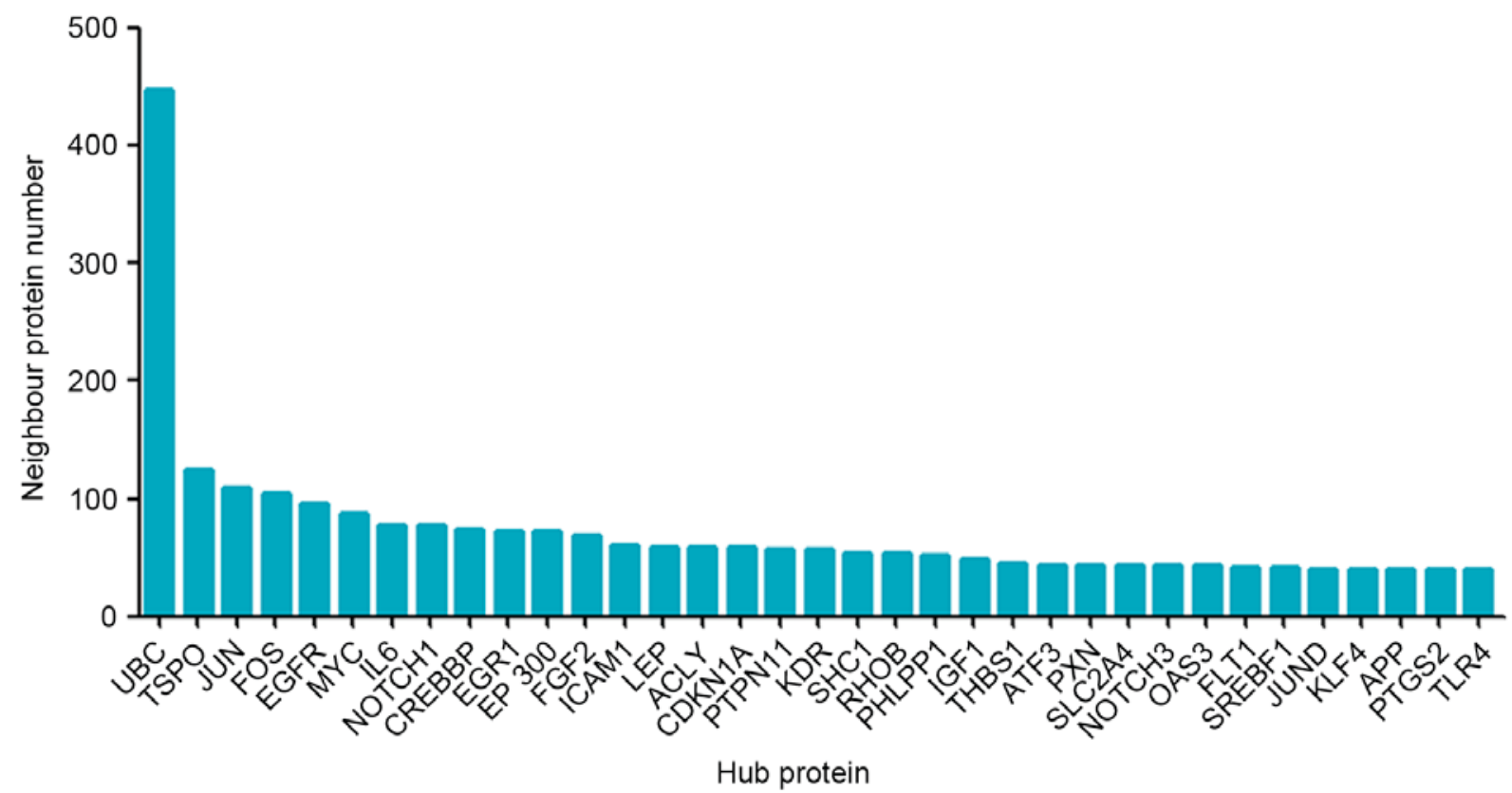

Figure 5. Protein-protein interaction analysis of these differentially expressed genes. Top 35 interacted proteins. The proteins directly linked with more than 40 other proteins were sequenced and the numbers of direct neighbors are presented.

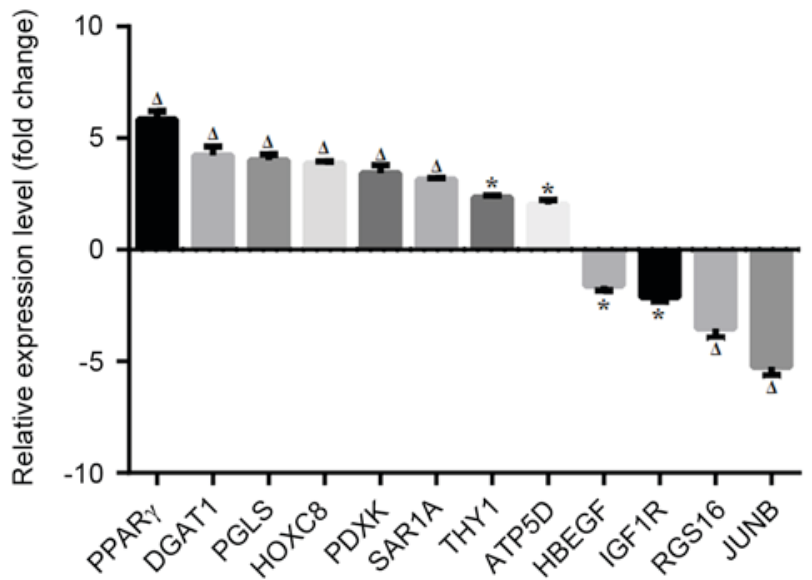

Figure 6. Validation of differentially expressed genes using RT-qPCR. MSL tissue specimens were subjected to RNA isolation and RT-qPCR amplification of randomly selected 12 differentially expressed genes and GAPDH was used for an endogenous control. Data collected from 3 independent experiments are presented as means \pm standard deviation; ${ }^{*} \mathrm{P}<0.05,{ }^{\Delta} \mathrm{P}<0.01$ vs. normal control tissues. MSL, multiple symmetric lipomatosis; RT-qPCR, reverse transcription-polymerase chain reaction; PPAR- $\gamma$, peroxisome proliferator activated receptor; PDXK, pyridoxal pyridoxine, vitamin B6 kinase; PGLS, phosphogluconolactonase; DGAT1, diacylglycerol O-acyltransferase 1; HOXC8, homeobox C8; ATP5D, ATP synthase, $\mathrm{H}^{+}$transporting, mitochondrial F1 complex, $\Delta$ Subunit A; THY-1, Thy-1 cell surface antigen; SAR1A, secretion associated Ras Related GTPase 1A; IGF1R, insulin like growth factor 1 receptor, HBEGF, heparin binding EGF like growth factor; RGS16, regulator of G-protein signaling 16; JUNB, Jun Proto-Oncogene, AP-1 Transcription Factor B.

UBC as the hub of the network was downregulated in MSL, suggesting that its downregulation may induce aberrant fat distribution by decreasing the ubiquitinated proteins that are involved in the adipocyte proliferation and adipogenesis, and that UBC may be a critical target in future control of MSL pathogenesis.
Another protein identified by the protein-protein interaction analysis was $18 \mathrm{kDa}$ TSPO, which formed a 124-protein network as direct neighbors (35). TSPO is known to be involved in cholesterol metabolism, oxidative stress and cardiovascular pathology, and in various human diseases, including metastatic cancer, Alzheimer's and Parkinson's diseases, inflammation and cardiovascular disorders (36-40). A previous study demonstrated that chronic high fat and cholesterol diet reduced the TSPO binding capacity in rat livers and aortas, suggesting that TSPO may be a compensatory responder to the challenges of the high-fat, high-cholesterol diet as a protection mechanism against the triggering of cell death $(41,42)$. Therefore, TSPO may be targeted as a novel strategy to reduce atherosclerosis risk or control atherosclerosis, although thus far, there are no studies associating TSPO with MSL.

Certain differentially expressed genes in MSL appeared to be from the same family of genes, including JUN, JUNB and JUND, or FOS, FOSB and FOSL2. Previous studies have reported that, when overexpressed, these gene families impair adipocyte differentiation and regulate adipogenesis through CCAAT/enhancer binding protein $(\mathrm{C} / \mathrm{EBP})-\alpha$ or $\mathrm{C} / \mathrm{EBP} \beta$ expression to differentiate mesenchymal cells into adipocytes (43-45). In the present study, levels of the transcription factors JUN, JUNB, JUND, FOS, FOSB and FOSL2 were decreased, whereas certain other critical regulatory genes of adipogenesis and lipid metabolism, including PPAR $\gamma$, sterol regulatory element binding transcription factor $1, \mathrm{C} / \mathrm{EBP} \alpha$, $\mathrm{C} / \mathrm{EBP} \beta$, insulin induced gene $1, \mathrm{ELOVL}$ fatty acid elongase 6 and LEP were significantly increased in MSL adipose tissue compared with normal controls. These data suggest that altered expression of these genes may participate in dysregulated adipocyte differentiation, leading to MSL development and progression.

In conclusion, the present study used differential gene expression analysis techniques to profile differentially 
expressed genes in MSL. A group of differentially expressed genes were identified in MSL, which exhibit the ability to alter adipocyte differentiation, lipid metabolism or protein ubiquitination, and therefore contribute to MSL development and progression. Future studies may be required to verify these differentially expressed genes using a larger spread of MSL tissue samples and explore the role of each differentially expressed gene and any underlying mechanisms associated with the development and progression of MSL.

\section{Acknowledgements}

The authors would like to thank Medjaden Bioscience Limited (Hong Kong, China) for assistance in editing of this manuscript.

\section{References}

1. Brodie BC (ed): Lecture XIV: On Fatty or Adipose Tumors In: Lectures Illustrative of Various Subjects In Pathology and Surgery. Longman, Brown, Green and Longmans, London, pp275-282, 1846.

2. Nisi G and Sisti A: Images in clinical medicine. Madelung's disease. N Engl J Med 374: 572, 2016.

3. Rendina D, De Filippo G, Nazzaro A and Strazzullo P: Impaired gonadal function in a woman with multiple symmetric lipomatosis. Minerva Endocrinol 38: 211-215, 2013.

4. Palacios E, Neitzschman HR and Nguyen J: Madelung disease: Multiple symmetric lipomatosis. Ear Nose Throat J 93: 94-96, 2014.

5. Esteban Júlvez L, Perelló Aragonés S and Aguilar Bargalló X: Sleep apnea-hypopnea syndrome and multiple symmetrical lipomatosis. Arch Bronconeumol 49: 86-87, 2013 (In English, Spanish).

6. Ardeleanu V, Chicos S, Georgescu C and Tutunaru D: Multiple benign symmetric lipomatosis-a differential diagnosis of obesity. Chirurgia (Bucur) 108: 580-583, 2013.

7. Yeh NC, Yang CY, Chou CW, Yen FC, Lee SY and Tien KJ: Madelung's disease. J Clin Endocrinol Metab 97: 3012-3013, 2012.

8. Tadisina KK, Mlynek KS, Hwang LK, Riazi H, Papay FA and Zins JE: Syndromic lipomatosis of the head and neck: A review of the literature. Aesthetic Plast Surg 39: 440-448, 2015.

9. Haap M, Siewecke C, Thamer C, Machann J, Schick F, Häring HU, Szeimies RM and Stumvoll M: Multiple symmetric lipomatosis: A paradigm of metabolically innocent obesity? Diabetes Care 27: 794-795, 2004.

10. Chen K, Xie Y, Hu P, Zhao S and Mo Z: Multiple Symmetric Lipomatosis: Substantial subcutaneous adipose tissue accumulation did not induce glucose and lipid metabolism dysfunction. Ann Nutr Metab 57: 68-73, 2010.

11. Cojocaru DC, Cozma CD and Postolache P: Markers of insulin resistance in a case of Launois-Bensaude syndrome. Rev Med Chir Soc Med Nat Iasi 117: 404-408, 2013.

12. Brea-García B, Cameselle-Teijeiro J, Couto-González I, Taboada-Suárez A and González-Âlvarez E: Madelung's disease: Comorbidities, fatty mass distribution and response to treatment of 22 patients. Aesthetic Plast Surg 37: 409-416, 2013.

13. Heike Z, Gudrun UM, Frank RD, Vetter H and Walger P: Multiple benign symmetric lipomatosis-a differential diagnosis of obesity: Is there a rationale for fibrate treatment? Obes Surg 18: 240-242, 2008.

14. Verhelle NA, Nizet JL, Van den Hof B, Guelinckx P and Heymans O: Liposuction in benign symmetric lipomatosis: Sense or senseless? Aesthetic Plast Surg 27: 319-321, 2003.

15. Enzi G, Busetto L, Sergi G, Coin A, Inelmen EM, Vindigni V, Bassetto F and Cinti S: Multiple symmetric lipomatosis: A rare disease and its possible links to brown adipose tissue. Nutr Metab Cardiovasc Dis 25: 347-353, 2015.

16. Ballester Sánchez R, Navarro Mira MÁ, Pujol Marco C and Botella Estrada R: Symmetric benign lipomatosis: Madelung syndrome. Med Clin (Barc) 141: 366-367, 2013 (In Spanish).

17. Kobayashi J, Nagao M, Miyamoto K and Matsubara S: MERRF syndrome presenting with multiple symmetric lipomatosis in a Japanese patient. Intern Med 49: 479-482, 2010.
18. Schoffer K and Grant I: Multiple lipomas, alcoholism, and neuropathy: Madelung's disease or MERRF? Muscle Nerve 33: 142-146, 2006.

19. Nisoli E, Regianini L, Briscini L, Bulbarelli A, Busetto L, Coin A, Enzi G and Carruba MO: Multiple symmetric lipomatosis may be the consequence of defective noradrenergic modulation of proliferation and differentiation of brown fat cells. J Pathol 198: 378-387, 2002.

20. Plummer C, Spring PJ, Marotta R, Chin J, Taylor G, Sharpe D, Athanasou NA, Thyagarajan D and Berkovic SF: Multiple symmetrical lipomatosis-a mitochondrial disorder of brown fat. Mitochondrion 13: 269-276, 2013.

21. Prantl L, Schreml J, Gehmert S, Klein S, Bai X, Zeitler K, Schreml S, Alt E, Gehmert S and Felthaus O: Transcription profile in sporadic multiple symmetric lipomatosis reveals differential expression at the level of adipose tissue-derived stem cells. Plast Reconstr Surg 137: 1181-1190, 2016.

22. Chen K, He H, Xie Y, Zhao L, Zhao S, Wan X, Yang W and Mo Z: miR-125a-3p and miR-483-5p promote adipogenesis via suppressing the RhoA/ROCK1/ERK1/2 pathway in multiple symmetric lipomatosis. Sci Rep 5: 11909, 2015.

23. Wang L, Feng Z, Wang X, Wang X and Zhang X: DEGseq: An Rpackage for identifying differentially expressed genes from RNA-seq data. Bioinformatics 26: 136-138, 2010.

24. Benjamini $\mathrm{Y}$ and HochbergY: Controlling the false discovery rate: A practical and powerful approach to multipletesting. J R Statist Soc 57: 289-300, 1995.

25. Mao X, Cai T, Olyarchuk JG and Wei L: Automated genome annotation and pathway identification using the KEGG Orthology (KO) as a controlled vocabulary. Bioinformatics 21: 3787-3793, 2005.

26. Kanehisa M, Araki M, Goto S, Hattori M, Hirakawa M, Itoh M, Katayama T, Kawashima S, Okuda S, Tokimatsu T and Yamanishi Y: KEGG for linking genomes to life and the environment. Nucleic Acids Res 36 (Database Issue): D480-D484, 2008.

27. Livak KJ and Schmittgen TD: Analysis of relative gene expression data using real-time quantitative PCR and the 2(-Delta Delta C(T)) method. Methods 25: 402-408, 2001.

28. Harsch IA, Bergmann T, Koebnick C, Wiedmann R, Ruderich F, Hahn EG and Konturek PC: Adiponectin, resistin and subclinical inflammation-the metabolic burden in Launois Bensaude Syndrome, a rare form of obesity. J Physiol Pharmacol 58 (Suppl 1): S65-S76, 2007.

29. Ouchi N: Adipocytokines in cardiovascular and metabolic diseases. J Atheroscler Thromb 23: 645-654, 2016.

30. Matsuzawa Y: Therapy insight: Adipocytokines in metabolic syndrome and related cardiovascular disease. Nat Clin Pract Cardiovasc Med 3: 35-42, 2006.

31. Ryu KY, Maehr R, Gilchrist CA, Long MA, Bouley DM, Mueller B, Ploegh HL and Kopito RR: The mouse polyubiquitin gene $\mathrm{UbC}$ is essential for fetal liver development, cell-cycle progression and stress tolerance. EMBO J 26: 2693-2706, 2007.

32. Dikic I, Wakatsuki S and Walters KJ: Ubiquitin-binding domains-from structures to functions. Nat Rev Mol Cell Biol 10: 659-671, 2009.

33. Ciechanover A: Intracellular protein degradation: From a vague idea through the lysosome and the ubiquitin-proteasome system and onto human diseases and drug targeting. Neurodegener Dis 10: 7-22, 2012.

34. Wójcik C, Lohe K, Kuang C, Xiao Y, Jouni Z and Poels E: Modulation of adipocyte differentiation by omega- 3 polyunsaturated fatty acids involves the ubiquitin-proteasome system. J Cell Mol Med 18: 590-599, 2014.

35. Santoro A, Mattace Raso G, Taliani S, Da Pozzo E, Simorini F, Costa B, Martini C, Laneri S, Sacchi A, Cosimelli B, et al: TSPO-ligands prevent oxidative damage and inflammatory response in C6 glioma cells by neurosteroid synthesis. Eur J Pharm Sci 88: 124-131, 2016.

36. Wyatt SK, Manning HC, Bai M, Ehtesham M, Mapara KY, Thompson RC and Bornhop DJ: Preclinical molecular imaging of the translocator protein (TSPO) in a metastases model based on breast cancer xenografts propagated in the murine brain. Curr Mol Med 12: 458-466, 2012.

37. Repalli J: Translocator protein (TSPO) role in aging and Alzheimer's disease. Curr Aging Sci 7: 168-175, 2014.

38. Cumming $\mathrm{P}$ and Borghammer P: Molecular imaging and the neuropathologies of Parkinson's disease. Curr Top Behav Neurosci 11: 117-148, 2012.

39. Qi X, Xu J, Wang F and Xiao J: Translocator protein (18 kDa): A promising therapeutic target and diagnostic tool for cardiovascular diseases. Oxid Med Cell Longev 2012: 162934, 2012. 
40. Li F, Liu J, Liu N, Kuhn LA, Garavito RM and Ferguson-Miller S: Protein $18 \mathrm{kDa}$ (TSPO): An Old protein with new functions? Biochemistry 55: 2821-2831, 2016.

41. Dimitrova-Shumkovska J, Veenman L, Ristoski T, Leschiner S and Gavish M: Chronic high fat, high cholesterol supplementation decreases $18 \mathrm{kDa}$ translocator protein binding capacity in association with increased oxidative stress in rat liver and aorta. Food Chem Toxicol 48: 910-921, 2010.

42. Lecanu L, Yao ZX, McCourty A, Sidahmed el-K, Orellana ME, Burnier MN and Papadopoulos V: Control of hypercholesterolemia and atherosclerosis using the cholesterol recognition/interaction amino acid sequence of the translocator protein TSPO. Steroids 78: 137-146, 2013.
43. Wagner EF and Eferl R: Fos/AP-1 proteins in bone and the immune system. Immunol Rev 208: 126-140, 2005.

44. Kveiborg M, Sabatakos G, Chiusaroli R, Wu M, Philbrick WM, Horne WC and Baron R: DeltaFosB induces osteosclerosis and decreases adipogenesis by two independent cell-autonomous mechanisms. Mol Cell Biol 24: 2820-2830, 2004.

45. Luther J, Driessler F, Megges M, Hess A, Herbort B, Mandic V, Zaiss MM, Reichardt A, Zech C, Tuckermann JP, et al: Elevated Fra-1 expression causes severe lipodystrophy. J Cell Sci 124: $1465-1476,2011$. 